\title{
Imaging Molecular Reaction and Diffusion in Organic Aerosol Particles
}

$\underline{\text { Peter A. Alpert }}^{1, *}$, Pablo Corral Arroyo ${ }^{1}$, Jing Dou ${ }^{2}$, Ulrich K. Krieger ${ }^{2}$, Sarah S. Steimer ${ }^{3}$, Jan-David Förster $^{4}$, Florian Ditas ${ }^{4}$, Christopher Pöhlker ${ }^{4}$, Stéphanie Rossignol ${ }^{5}$, Monica Passananti ${ }^{6}$, Sebastian Perrier $^{7}$, Christian George ${ }^{7}$, Thomas Berkemeier ${ }^{8}$, Manabu Shiraiwa ${ }^{9}$ and Markus Ammann .

1. Laboratory of Environmental Chemistry, Paul Scherrer Institute, 5232 Villigen, Switzerland.

2. Institute for Atmospheric and Climate Science, ETH Zürich, 8092 Zürich, Switzerland

3. Department of Chemistry, University of Cambridge, Cambridge, CB2 1EW, United Kingdom

4. Multiphase Chemistry Department, Max Planck Institute for Chemistry, 55128 Mainz, Germany

5. Aix Marseille Université, CNRS, LCE UMR 7376, 13331 Marseille, France

6. Department of Physics, University of Helsinki, 00014 Helsinki, Finland

7. Université Lyon 1, CNRS, UMR 5256, IRCELYON, 69626 Villeurbanne, France

8. School of Chemical \& Biomolecular Engineering, Georgia Institute of Technology, Atlanta, GA 30332, United States

9. Department of Chemistry, University of California, Irvine, CA 92617, United States

* Corresponding author, peter.alpert@psi.ch.

Atmospheric aerosol particles are composed of water and a variety of inorganic and organic components stemming from natural and anthropogenic sources [1]. Airborne organic matter, in particular, can be in a highly viscous or glassy phase with properties comparable to that of a solid. It was found that water, the atmospheric solvent, diffuses through a glassy organic particle quite slowly and establishes equilibrium with relative humidity only after timescales on the order of days [2]. In addition to water, diffusion of oxidizing trace gas molecules such as $\mathrm{O}_{3}$ through highly viscous organic aerosol can be limited and may lead to an inhomogeneous $\mathrm{O}_{3}$ concentration and reduced chemical reaction rates compared to what is expected when assuming a homogeneous mixture [3,4]. Reaction and diffusion kinetics of $\mathrm{O}_{3}$ with organic shikimic acid particles was quantified in viscous organic matter using X-ray microspectroscopy however, any concentration gradients could not be visualized on spatial scale of tens of nanometers [3]. Visualizing chemical gradients due to gas-to-particle reaction would allow direct evidence of coupled molecular diffusion and reaction in aerosol particles for the first time, which we find necessary for accurate predictions of atmospheric aerosol chemical aging.

We present a study using scanning transmission X-ray microscopy coupled to near edge X-ray absorption fine structure spectroscopy (STXM/NEXAFS) to visualize the reaction of $\mathrm{O}_{3}$ with iron to change its oxidation state in a non-reactive organic matrix, Xanthan gum, a model material for organic/biogenic marine derived particles aerosolized from the ocean surface [5]. Aerosol particles with equal mass ratio of Xanthan gum and $\mathrm{FeCl}_{2}$ were situated in a custom environmental cell having diameters, $d_{p}$, between $0.2-2 \mu \mathrm{m}$. We exposed particles to $\mathrm{O}_{3}$ at $150 \mathrm{ppb}$, relative humidity (RH) at $40 \%$, $\mathrm{O}_{2}$ at 40 mbar and $\mathrm{He}$ at 110 mbar while observing X-ray absorption at the Fe L-edge in situ. Two absorption peaks at energies corresponding to $\mathrm{Fe}^{\mathrm{II}}$ and $\mathrm{Fe}^{\mathrm{III}}$ were mapped with $35 \mathrm{x} 35 \mathrm{~nm}$ spatial resolution and used to derive the fraction of $\mathrm{Fe}^{\mathrm{II}}, \alpha$, [6] a tracer for $\mathrm{O}_{3}$ reaction and diffusion. A kinetic multi-layer model, KM-SUB [4], was used to simulate our experiment and reproduce internal profiles of $\alpha$ using fit parameters which were Henry's law coefficient, $H$, and $\mathrm{O}_{3}$ and Fe diffusion coefficients, $D_{O_{3}}$ and $D_{\mathrm{Fe}}$, respectively, in the Xanthan gum matrix. The reaction rate coefficient of $\mathrm{O}_{3}$ with $\mathrm{Fe}^{\mathrm{II}}$ in aqueous solution was $k=8 \cdot 10^{-16} \mathrm{~cm}^{-3} \mathrm{~s}^{-1}$ from NIST Chemical Kinetics Database found at 
http://kinetics.nist.gov/solution.

Figure 1a shows that $\alpha$ decreased from about 0.9 to 0.5 over the course of 4 hours. Values of $\alpha$ were also averaged at each $t$ over concentric pixels from particle perimeters thus deriving 2-D vertically integrated profiles. Examples are shown in Fig. $1 \mathrm{~b}$ and reveal that $\alpha$ is less at particle surfaces than at particle interiors. Model results from KM-SUB are shown which capture the general trend of $\mathrm{O}_{3}$ reaction kinetics and corresponding chemical profiles, where $H=5 \cdot 10^{-5} \mathrm{~mol} \mathrm{~cm} \mathrm{~atm}^{-}$ ${ }^{1}, D_{O_{3}}=3 \cdot 10^{-6} \mathrm{~cm}^{2} \mathrm{~s}^{-1}$ and $D_{\mathrm{Fe}}=3$. $10^{-14} \mathrm{~cm}^{2} \mathrm{~s}^{-1}$. The latter value is typical for a semi-solid material which is close in viscosity to putty. However, $D_{O_{3}}$ is quite high and comparable to water selfdiffusion. More data sets at higher and lower humidity should be included to better

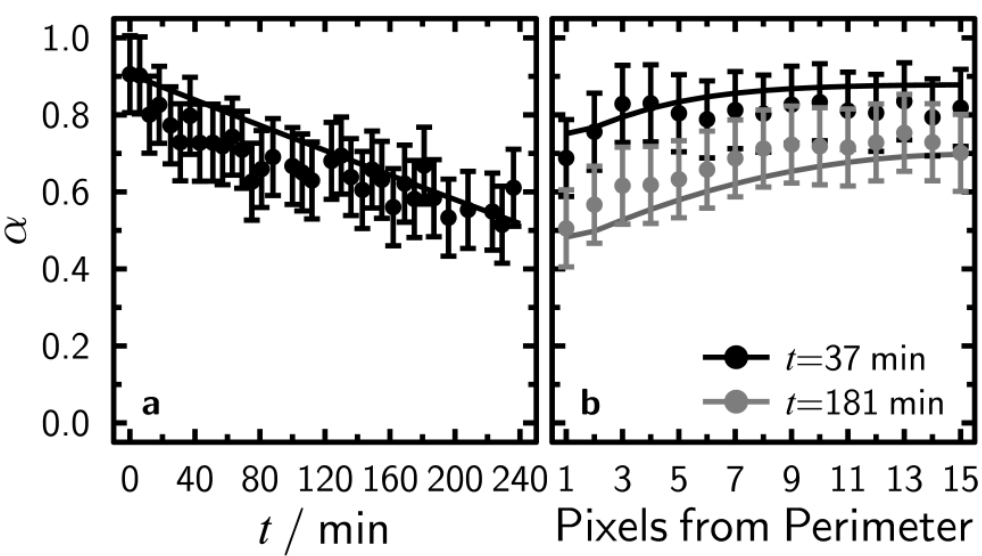

Figure. 1. (a) Average $\mathrm{Fe}^{\mathrm{II}}$ fraction, $\alpha$, as a function of time, $t$. Error bars in $\alpha$ are \pm 0.1 and calculated from the particle to particle standard deviation from the respective mean. Each data point represents the average of $\sim 10$ particles. (b) The 2-D projected profile of $\boldsymbol{\alpha}$ as a function of the pixels from particle perimeters. A single profile corresponds to a single data point in $t$ (indicated in the legend) included in panel (b). Error bars are taken from panel (a). Model results are shown as solid lines. constrain these fit parameters. A 3-D gradient in $\alpha$ evolving over time must have existed in order to explain our results, which could only have developed due to coupled reaction and diffusion of Fe and $\mathrm{O}_{3}$. We calculate the reacto-diffusive length, $l$, a length scale indicating the distance that $\mathrm{O}_{3}$ molecules traveled until they reacted. Under our conditions, $l=\sqrt{D_{O_{3}} /\left(k C_{F e^{I I}}\right)}=9 \mathrm{~nm}$, where $C_{F e^{I I}}=5 \cdot 10^{21}$ $\mathrm{cm}^{-3} \mathrm{~s}^{-1}$ was the concentration of $\mathrm{Fe}^{\mathrm{II}}$. In other words, $\mathrm{O}_{3}$ must have only penetrated tens of nanometers into the particle surface, but was completely absent in the core. If so, the observed chemical gradients must have been largely due to $\mathrm{Fe}^{\mathrm{II}}$ and $\mathrm{Fe}^{\mathrm{III}}$ diffusing throughout the particle. We emphasize that the $e$ folding time for $\mathrm{O}_{3}$ to saturate a particle with $d_{p}=1 \mu \mathrm{m}$ is $\tau=d_{p} /\left(4 \pi D_{O_{3}}\right)$ or about 3 seconds when neglecting reaction. Therefore, we strongly suggest that prediction of aerosol chemistry must consider a coupled reacto-diffusive process, which we have observed can result in an inhomogeneous internal concentration gradient over timescales of hours. However, this is highly dependent on the balance between diffusion, reaction rate and concentration of reactants in the condensed phase [7].

\section{References:}

[1] A. Laskin et al, Annu. Rev. Anal. Chem. 9 (2016), p. 117-143.

[2] M. Shiraiwa et al, Nat. Comm. 8 (2017), 15002.

[3] S. S. Steimer et al, Atmos. Chem. Phys. 14 (2014), 10761-10772.

[4] T. Berkemeier et al, Phys. Chem. Chem. Phys. 18 (2016), 12662-12674.

[5] P. A. Alpert et al, J. Geophys. Res. Atmos. 14 (2015), 8841-8860.

[6] R. C. Moffet et al, J. Geophys. Res. 14 (2012), D07204.

[7] The authors acknowledge the Swiss National Science Foundation grant no. 163074, the European Union's Horizon 2020/Marie Skłodowska-Curie grant agreement no. 701647 and B. Watts, who was crucial in supporting the operation of our experimental infrastructure at the PolLux beamline. 